\title{
Impact of lymphopenia on survival for elderly patients with glioblastoma: A secondary analysis of the CCTG CE.6 (EORTC 26062-22061, TROG08.02) randomized clinical trial
}

\author{
Andrew J. Song, Keyue Ding, lyad Alnahhas, Normand J. Laperriere, James Perry, Warren P. Mason, \\ Chad Winch, Chris J. O'Callaghan, Johan J. Menten, Alba A. Brandes, Claire Phillips, Michael F. Fay,

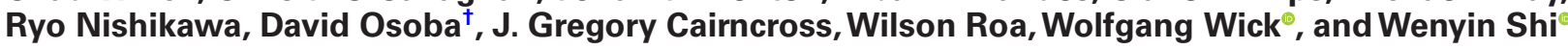

\begin{abstract}
Department of Radiation Oncology, Thomas Jefferson University, Philadelphia, Pennsylvania, USA (A.J.S., W.S.); Department of Neurological Surgery, Thomas Jefferson University, Philadelphia, Pennsylvania, USA (I.A.); Department of Public Health Sciences, Canadian CancerTrials Group, Queen's University, Kingston, Ontario, Canada (K.D., C.W., C.J.O.); Department of Radiation Oncology, Princess Margaret Cancer Center, Toronto, Ontario, Canada (N.J.L.); Department of Medicine, Division of Neurology, University of Toronto, Toronto, Ontario, Canada (J.P.); Department of Medicine, Princess Margaret Cancer Centre, University of Toronto, Toronto, Ontario, Canada (W.P.M.); Department of Experimental Radiation Oncology, University Hospitals Leuven, Leuven, Belgium (J.J.M.); Department of Medical oncology, IRCCS Istituto Scienze Neurologiche - Bologna, Italy (A.A.B.); Department of Radiation Oncology, Peter MacCallum Cancer Centre, Melbourne, Australia (C.P.); Genesis Cancer Care, Newcastle, NSW, Australia (M.F.); Department of Neuro-Oncology/Neurosurgery, Saitama Medical University International Medical Center, Hidaka, Japan (R.N.); OOL Consulting, West Vancouver, British Columbia, Canada (D.O.); Department of Clinical Neurosciences, University of Calgary, Calgary, Alberta, Canada (J.G.C.); Division of Radiation Oncology, Cross Cancer Institute, Edmonton, Alberta, Canada (W.R.); Division of Neurology, Heidelberg University Medical Center, Clinical Cooperation Unit Neurooncology, German Cancer Research Center, Heidelberg, Germany (W.W.)
\end{abstract}

Corresponding Author: Wenyin Shi, MD, PhD, Department of Radiation Oncology, Thomas Jefferson University, 111 S 11th St, G-301, Philadelphia, PA 19107, USA (Wenyin.Shi@ Jefferson.edu).

${ }^{\dagger}$ Deceased.

Abstract

Background. Lymphopenia may lead to worse outcomes for glioblastoma patients. This study is a secondary analysis of the CCTG CE.6 trial evaluating the impact of chemotherapy and radiation on lymphopenia, and effects of lymphopenia on overall survival (OS).

Methods. CCTG CE. 6 randomized elderly glioblastoma patients ( $\geq 65$ years) to short-course radiation alone (RT) or short-course radiation with temozolomide (RT +TMZ). Lymphopenia (mild-moderate: grade 1-2; severe: grade 3-4) was defined per CTCAE v3.0, and measured at baseline, 1 week and 4 weeks post-RT. Preselected key factors for analysis included age, sex, ECOG, resection extent, MGMT methylation, Mini-Mental State Examination, and steroid use. Multinomial logistic regression and multivariable Cox regression models were used to identify lymphopenia-associated factors and association with survival.

Results. Five hundred and sixty-two patients were analyzed (281 RT vs $281 \mathrm{RT}+\mathrm{TMZ}$ ). At baseline, both arms had similar rates of mild-moderate (21.4\% vs $21.4 \%$ ) and severe (3.2\% vs $2.9 \%)$ lymphopenia. However, at 4 weeks post-RT, RT+TMZ was more likely to develop lymphopenia (mild-moderate: $27.9 \%$ vs $18.2 \%$; severe: $9.3 \%$ vs $1.8 \%$; p<0.001). Developing any lymphopenia post-RT was associated with baseline lymphopenia $(P<.001)$. Baseline lymphopenia (hazard ratio [HR] 1.3$)$ was associated with worse OS (HR: 1.30, 95\% confidence interval $[\mathrm{Cl}] 1.05-1.62 ; P=.02$ ), regardless of MGMT status.

Conclusions. Development of post-RT Iymphopenia is associated with addition of TMZ and baseline lymphopenia and not with RT alone in patients treated with short-course radiation. However, regardless of MGMT status, only 
baseline lymphopenia is associated with worse OS, which may be considered as a prognostic biomarker for elderly glioblastoma patients.

\section{Key Points}

- This is a secondary analysis of the CCTG CE.6 trial evaluating the impact of lymphopenia.

- Development of lymphopenia is not associated with RT alone.

- Baseline lymphopenia is associated with worse OS.

\section{Importance of the Study}

This is a secondary analysis of CCTG CE. 6 trial evaluating the impact of lymphopenia with overall survival in elderly patients with glioblastoma. The study demonstrated that the hypofractionated radiation treatment regimen used in the trial did not lead to development of lymphopenia. Rather, development of postradiation lymphopenia was associated with addition of $\mathrm{TMZ}$ and baseline lymphopenia. Regardless of MGMT status, only baseline lymphopenia is associated with worse OS, which may be considered as a prognostic biomarker for elderly glioblastoma patients. This is the first study evaluated the incidence of treatment developed lymphopenia with hypofractionated radiation with and without temozolomide, as well as the impact of Iymphopenia on overall survival of elderly patients with newly diagnosed glioblastoma based on prospectively collected data.
Advancing the treatment paradigm for elderly glioblastoma patients has been a complex balancing act between maximizing survival and aggressiveness of care. Compared to best supportive care, adjuvant radiation therapy (RT) with conventional fractionation (standard course) was shown to nearly double median survival times (16.9 weeks vs 29.1 weeks) without compromising quality of life (OOL). ${ }^{1}$ Additional studies explored hypofractionation, with various shortened treatment schedules showing no difference compared to outcomes with conventional fractionation. ${ }^{2,3}$ Temozolomide alone was found to be comparable for survival to standard course alone ${ }^{4}$ and hypofractionated radiation alone. ${ }^{5}$

Canadian Cancer Trials Group (CCTG) CE.6 (EORTC 26062-22061, TROG08.02) was a multi-institutional, openlabel randomized Phase III clinical trial investigating the benefits of adding temozolomide to hypofractionated (short course) RT for elderly (age $\geq 65$ years) patients with newly diagnosed glioblastoma. ${ }^{6}$ The authors reported both an overall survival (OS) and progression-free survival (PFS) benefit, with a more pronounced effect in patients with methylated $\mathrm{O}^{6}$-methylguanine-DNA methyltransferase (MGMT) status. There is concern, however, that the addition of temozolomide to radiation may lead to increased incidence and severity of lymphopenia after concurrent treatment has been completed.

Lymphopenia has been observed in glioblastoma patients receiving standard of care (SOC) chemoradiation, with up to $40 \%$ developing grade 3 or higher adverse events. ${ }^{7}$ Temozolomide has demonstrated a dose-dependent toxicity for Iymphopenia in glioblastoma. ${ }^{8}$ Glioblastoma patients are also oftentimes exposed to steroids, which also exhibit a dose-dependent association with lymphopenia. ${ }^{9}$ Lymphopenia, particularly with a severe reduction in lymphocyte counts, has been associated with worse survival outcomes, with a hazard ratio $(\mathrm{HR})$ of 1.8 for malignant gliomas receiving SOC, resulting in a median OS difference of 16 versus 18 months. ${ }^{10}$ Elderly patients who are predisposed to being immunocompromised ${ }^{11}$ may be at higher risk for this phenomenon.

To date, there has not been an analysis of prospectively collected data to compare incidence and severity of treatment-related lymphopenia (TRL) between the treatment arms of radiation alone (RT) versus radiation with temozolomide (RT $+\mathrm{TMZ}$ ). Based on these considerations, we performed a secondary analysis of CCTG CE. 6 to investigate the impact of concurrent chemoradiation versus RT alone on the incidence and severity of lymphopenia in this patient population and the potential association with survival outcomes.

\section{Methods}

This study was approved by the Institutional Review Board at Thomas Jefferson University (Philadelphia, PA, USA). The original study was registered on ClinicalTrials.gov (Identifier: NCT00482677). 


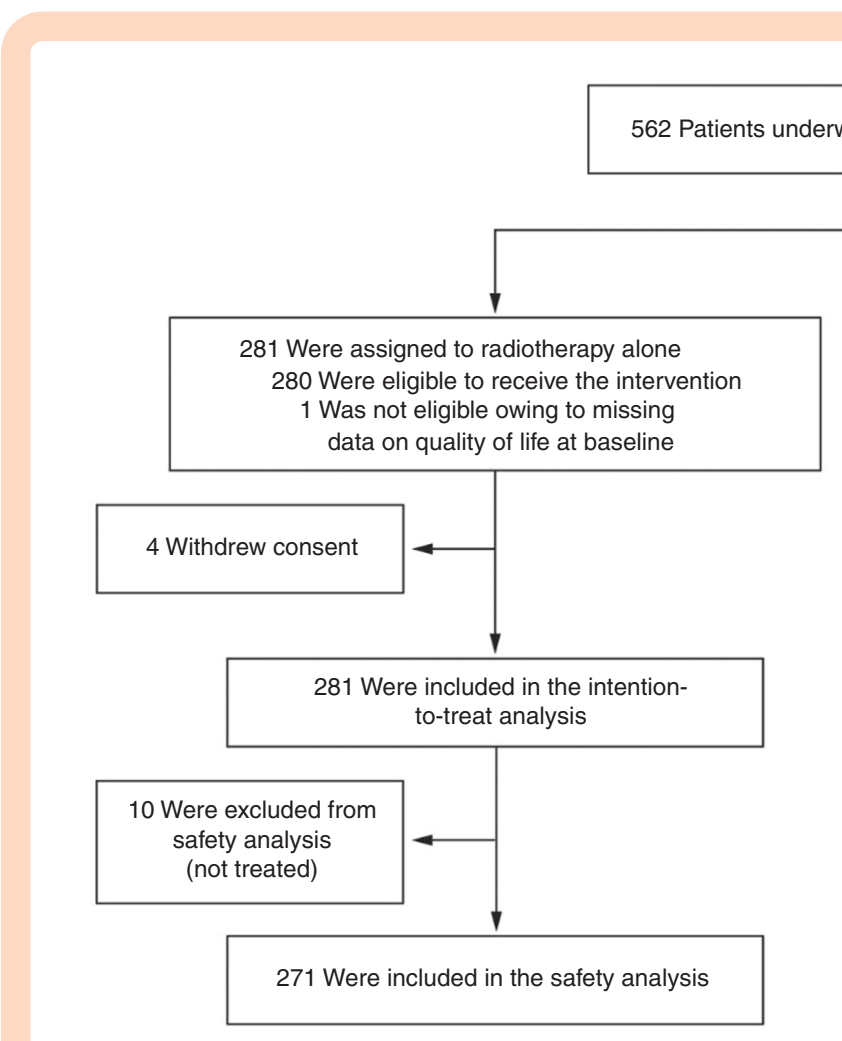

Figure 1. CONSORT diagram from CCTG CE.6.

\section{Patient Selection}

All eligible patients enrolled and randomized who received treatment in CCTG CE. 6 from November 2007 to September 2013 were included in this analysis. See CONSORT diagram from the original study in Figure 1. Treatment details for each arm, for example, radiation therapy and temozolomide dosing, are included in the original publication for reference. ${ }^{6}$ Patients who had evidence of disease progression or died prior to the time of 4 weeks after completion of RT, or missing hematology evaluation, were excluded from the analysis of week 4 lymphopenia's status on PFS and OS.

\section{Radiation Treatment}

Short-course radiation was delivered as $40.05 \mathrm{~Gy}$ in 15 fractions over 3 weeks. A single planning target volume (PTV) was derived from: (1) Gross tumor volume (GTV) defined as the contrast-enhancing volume on the postoperative planning magnetic resonance imaging (MRI) scan including surgical bed, (2) Clinical target volume (CTV) derived from GTV plus a $1.5-\mathrm{cm}$ margin respecting anatomical boundaries, and (3) PTV CTV plus margin of $0.5 \mathrm{~cm}$ was applied. 3D planning techniques were utilized, while intensity-modulated radiation therapy was not permitted as specified per clinical trial protocol.

\section{Temozolomide}

Concurrent temozolomide was administered with radiotherapy at a dose of $75 \mathrm{mg}$ per square meter of body-surface area per day for 21 consecutive days from day 1 until the final day of radiotherapy. Adjuvant temozolomide was administered at a dose of 150$200 \mathrm{mg}$ per square meter per day for 5 consecutive days of a 28-day cycle for up to 12 cycles or until disease progression.

\section{Lymphopenia Analysis}

Lymphopenia was defined per Common Terminology Criteria for Adverse Events (CTCAE) v3.0 (US Department of Health and Human Services, National Institutes of Health, National Cancer Institute) by the following grouping of grades $^{12}$ :

1) Mild-moderate [Grade 1: $<L L N \times 0.8-10^{9} / \mathrm{L}$ and Grade 2: $\left.<0.8-0.5 \times 10^{9} / \mathrm{L}\right]$

2) Severe [Grade 3: $<0.5-0.2 \times 10^{9} / \mathrm{L}$ and Grade 4 : $<0.2 \times$ $10 \%$ L]

Lymphocyte counts were collected at baseline and post-radiation, including at 1 week and 4 weeks after completion. 


\section{Statistical Analysis}

Preselected key baseline patient characteristics for the analysis included:

a) Age (65-70 years old; 71-75 years old; 76 years and older)

b) Sex (Male; Female)

c) ECOG Performance Status (0 or $1 ; 2$ )

d) Extent of resection (Biopsy only; partial or complete resection)

e) MGMT promoter methylation status (Methylated; Unmethylated)

f) Mini-Mental State Exam (0-30)

g) Steroid use at study randomization (Yes; No)

h) Assigned treatment arm (RT; RT+TMZ)

Chi-square test was used to assess association categorical variables; analysis of variance was used to test association of continuous factors with lymphopenia status; Kaplan-Meier curves were used to estimate distributions of the time to event outcomes. Landmark analysis was performed to study the association of week 4 lymphopenia's status on OS and PFS. Multinomial logistic regression models were used to identify factors associated with lymphopenia; and multivariable Cox regression models were then used to study effects of Iymphopenia across time intervals on survival outcomes while adjusting baseline factors. All reported $P$ values are 2 sided, and $P$ value $<$ .05 was considered statistically significant. Analyses were performed using SAS software (version 9.3; SAS Institute).

\section{Results}

\section{Patient Characteristics}

There was a total of 562 patients, of which 281 were in the $\mathrm{RT}$ alone arm and 281 received $\mathrm{RT}+\mathrm{TMZ}$. Of these, 74 patients (41 from RT and 33 from RT+TMZ) were excluded from survival analysis due to early progression or death, resulting in 488 patients ( $240 \mathrm{RT}$ and $248 \mathrm{RT}+\mathrm{TMZ}$ ). Patients who had early progression or death were more likely to have worse ECOG performance status $(P=.02)$, biopsy only $(P<.001)$, steroid use $(P=.04)$, and lower Mini-Mental State Examination (MMSE) $(P=.06)$.

Additional demographic and patient-related information can be found in Table 1.

\section{Lymphopenia Incidence and Severity}

At baseline, Iymphopenia rates were virtually identical between treatment arms, with approximately $75.6 \%$ of patients with normal lymphocyte counts, $21.4 \%$ with mild-moderate lymphopenia, and $3 \%$ with severe lymphopenia (Table 2). At 1 -week post-RT, there was a small numerical increase in mildmoderate lymphopenia for patients on $\mathrm{RT}+\mathrm{TMZ}(15.4 \%$ vs $10.7 \%)$, however, this was not statistically significant $(P=.25)$. At 4 weeks post-RT, there was a significant increase for both incidence and severity of lymphopenia for patients who received RT+TMZ versus RT, with $37.2 \%$ versus $20 \%$, of which
Table 1. Patient Characteristics

\begin{tabular}{|c|c|c|c|}
\hline Tab & Early P/D & $\begin{array}{l}\text { Non-Early } \\
\text { P/D }\end{array}$ & $P$-values \\
\hline Age (years) & $n(\%)$ & $n(\%)$ & .10 \\
\hline $65-70$ & $27(36.5)$ & $138(28.3)$ & \\
\hline $71-75$ & $22(29.7)$ & $209(42.8)$ & \\
\hline 76 and older & $25(33.8)$ & $141(28.9)$ & \\
\hline Sex & & & .83 \\
\hline Female & $28(37.8)$ & $191(39.1)$ & \\
\hline Male & $46(62.2)$ & $297(60.9)$ & \\
\hline ECOG Performance & & & .02 \\
\hline$\leq 2$ & $49(66.2)$ & $383(78.5)$ & \\
\hline 2 & $25(33.8)$ & $105(21.5)$ & \\
\hline Extent of Resection & & & $<.01$ \\
\hline Biopsy only & $35(47.3)$ & $131(26.8)$ & \\
\hline Partial or complete & $39(52.7)$ & $357(73.2)$ & \\
\hline Treatment Arm & & & .32 \\
\hline RT & $41(55.4)$ & $240(49.2)$ & \\
\hline $\mathrm{RT}+\mathrm{TMZ}$ & $33(44.6)$ & $248(50.8)$ & \\
\hline Steroids Use & & & .04 \\
\hline Yes & $63(85.1)$ & $360(73.8)$ & \\
\hline No & $11(14.9)$ & $128(26.2)$ & \\
\hline Lymphopenia (BL or $1 \mathrm{~W}$ ) & & & .82 \\
\hline Grade $\geq 1$ & $26(35.1)$ & $178(36.5)$ & \\
\hline Grade 0 & $48(64.9)$ & $310(63.5)$ & \\
\hline $\begin{array}{l}\text { MGMT methylation } \\
\text { status }\end{array}$ & & & .33 \\
\hline Methylated & $18(24.3)$ & $147(30.1)$ & \\
\hline Un methylated & $23(31.1)$ & $166(34.0)$ & \\
\hline Unknown & $33(44.6)$ & $175(35.9)$ & \\
\hline \multirow[t]{2}{*}{ MMSE } & $\begin{array}{l}\text { Mean } \\
\text { (STD) }\end{array}$ & Mean (STD) & .06 \\
\hline & $24.4(5.6)$ & $25.7(4.4)$ & \\
\hline
\end{tabular}

$B L$, baseline; 1W, lymphocyte count 1-week post-RT; P/D, progression or death; STD, standard deviation.

9.3\% versus $1.8 \%$ were Grade 3 or higher, respectively $(P<$ $.0001)$. The rate of lymphopenia at 4 weeks post-RT is significantly higher than 1 week post-RT in the RT-TMZ group, but not in the RT alone group.

At 4 weeks post-RT, factors associated with developing lymphopenia included treatment arm RT+TMZ (OR 2.15; 95\% Cl 1.38-3.33; $P=.0007$ ) and lymphopenia present at baseline (OR 5.12;95\% Cl 3.30-7.94; $P<.0001$ ). These factors were also associated with developing severe lymphopenia, with RT+TMZ (OR 7.64; 95\% Cl 2.79-20.94; $P<.0001$ ) and baseline lymphopenia (OR 9.01; 95\% Cl -3.80-21.38; $P<.0001$ ), as well as biopsy only patients (OR 2.56; 95\% Cl 1.16-5.65; $P=.02$ ). Age, sex, ECOG, MGMT status, steroid use at baseline, and MMSE were not found to be associated with lymphopenia at 4 weeks post-RT in multivariable analysis. 
Table 2. Lymphopenia by Treatment Arms at Different Time Intervals

\begin{tabular}{|c|c|c|c|c|c|}
\hline \multirow[t]{2}{*}{ Treatment Arm } & \multicolumn{4}{|c|}{$\underline{\text { Lymphopenia at Baseline }}$} & \\
\hline & Grade 0 & Grades $1 \& 2$ & Grades $3 \& 4$ & Total & \\
\hline RT & $212(75.4 \%)$ & $60(21.4 \%)$ & $9(3.2 \%)$ & 281 & \\
\hline $\mathrm{RT}+\mathrm{TMZ}$ & $213(75.8 \%)$ & $60(21.4 \%)$ & $8(2.9 \%)$ & 281 & \\
\hline \multirow[t]{2}{*}{ Treatment Arm } & \multicolumn{4}{|c|}{ Lymphopenia at post-RT 1 week } & \multirow[b]{2}{*}{$P$-value } \\
\hline & Grade 0 & Grades $1 \& 2$ & Grades $3 \& 4$ & Total & \\
\hline RT & $238(84.7 \%)$ & $30(10.7 \%)$ & $13(4.6 \%)$ & 281 & .25 \\
\hline $\mathrm{RT}+\mathrm{TMZ}$ & $224(80.0 \%)$ & $43(15.4 \%)$ & $13(4.6 \%)$ & 280 & \\
\hline \multirow[t]{2}{*}{ Treatment Arm } & \multicolumn{4}{|c|}{ Lymphopenia at post-RT 4 weeks } & \multirow[b]{2}{*}{$P$-value } \\
\hline & Grade 0 & Grades $1 \& 2$ & Grades $3 \& 4$ & Total & \\
\hline RT & $225(80.1 \%)$ & $51(18.2 \%)$ & $5(1.8 \%)$ & 281 & $<.0001$ \\
\hline $\mathrm{RT}+\mathrm{TMZ}$ & $176(62.9 \%)$ & $78(27.9 \%)$ & $26(9.3 \%)$ & 280 & \\
\hline
\end{tabular}

\section{Impact on OS and PFS}

OS was worse for patients who developed lymphopenia at 4 weeks post-RT (HR 1.29; 95\% Cl 1.04-1.60; $P=.02)$ with median OS of 7.95 months versus 9.92 months (Figure 2) in landmark analysis. Comparison across lymphopenia severity did not yield statistically significant differences between mild-moderate versus severe lymphopenia $(P=.07)$, with median of OS of 7.92 versus 8.08 months, respectively.

However, after adjusting for confounding factors on multivariable modeling, lymphopenia status at 4 weeks post-RT was no longer associated with OS $(P=.49)$, including for severe lymphopenia $(P=.58)$. Instead, baseline lymphopenia (HR 1.30; $P=.02$ ), males (HR 1.41), biopsy only (HR 1.59), and lower MMSE (HR 0.975), were significantly associated with worse OS (Table 3 ).

Similarly, 4-weeks post-RT lymphopenia was significantly associated with PFS on univariate (HR 1.25; $P=.04)$, but not in multivariate analysis $(P=.21)$. Baseline lymphopenia was marginally significant for worse PFS (HR 1.22; $P=.06$ ), while male patients (HR 1.27; $P=.01$ ), biopsy only (HR 1.33), and lower MMSE patients had significantly worse PFS.

On the other hand, patients with baseline lymphopenia have significantly worse OS $(P=.0037, \mathrm{HR} 1.341,95 \% \mathrm{Cl}$ 1.099-1.637), and PFS ( $P=.0356$, HR $1.235,95 \% \mathrm{Cl} 1.013-$ 1.506). This remains significant after adjusting for confounding factors on multivariate modeling (Figure 2).

\section{MGMT Promoter Methylation Status Stratification}

When stratifying survival analysis by MGMT promoter methylation status, baseline lymphopenia (HR 1.66; $P=.02)$, males (HR 1.45; $P=.04$ ), steroid use at baseline (HR 1.84; $P=.005$ ) were significantly associated with worse OS in MGMT methylated patients. Baseline lymphopenia (HR 1.59; $P=.004$ ) and lower MMSE (HR 0.961; $P=.05$ ) were significantly associated with worse OS in MGMT unmethylated patients.

For PFS, MGMT methylated patients who were male (HR1.61; $P=.01$ ) and steroid use (1.86; $P=.005)$, had worse outcomes. Baseline lymphopenia (HR 1.75; $P=.003$ ) and worse ECOG (HR 1.65; $P=.05$ ) were associated with reduced PFS in MGMT unmethylated patients.

\section{Discussion}

Our study results confirm our hypothesis that the addition of concurrent TMZ to RT results in not only increased incidence of treatment-related lymphopenia, but also increased severity. However, surprisingly, the severity and incidence of 4 weeks post-RT lymphopenia did not impact OS or PFS, including on subgroup analysis by MGMT methylation status. Instead, lymphopenia present at baseline of both mild-moderate and severe grades was significantly associated with OS for both MGMT methylated and unmethylated patients.

Our study is unique as the largest reported for exploring lymphocyte counts in glioblastoma patients from a prospectively conducted investigation. With a 1:1 split between treatment arms and all patients receiving short-course RT by clinical trial design, this provides a well-controlled environment answering questions on how specific treatment modalities may contribute to the incidence and severity of lymphopenia, and more importantly the impact these events may have on survival outcomes. Although our results may appear discordant with prior reports of smaller studies in the GBM literature, ${ }^{10,13}$ we believe that our finding on baseline lymphopenia as a prognostic factor may, in a way, is in agreement. ${ }^{14-16}$ Previously reported literature has various definitions for baseline lymphopenia, ranging from lymphocyte counts of $800 / \mu \mathrm{L}$ to $1000 / \mu \mathrm{L}$ and $1500 / \mu \mathrm{L} .{ }^{10,17-19}$ Studies specifically addressing this question are limited, with one publication from the literature addressing lymphopenia in elderly GBM, however, this was a retrospective study of 72 patients, of which just 20 patients $(30.56 \%)$ received short-course $\mathrm{RT} .{ }^{14}$ Thus, our study provides a unique prospective outlook on elderly GBM which typically has less aggressive RT and concurrent phase therapy.

Lymphopenia rates reported previously in primary as par for hematologic toxicity, but not stratified by timepoints and not statistically analyzed for significance between 


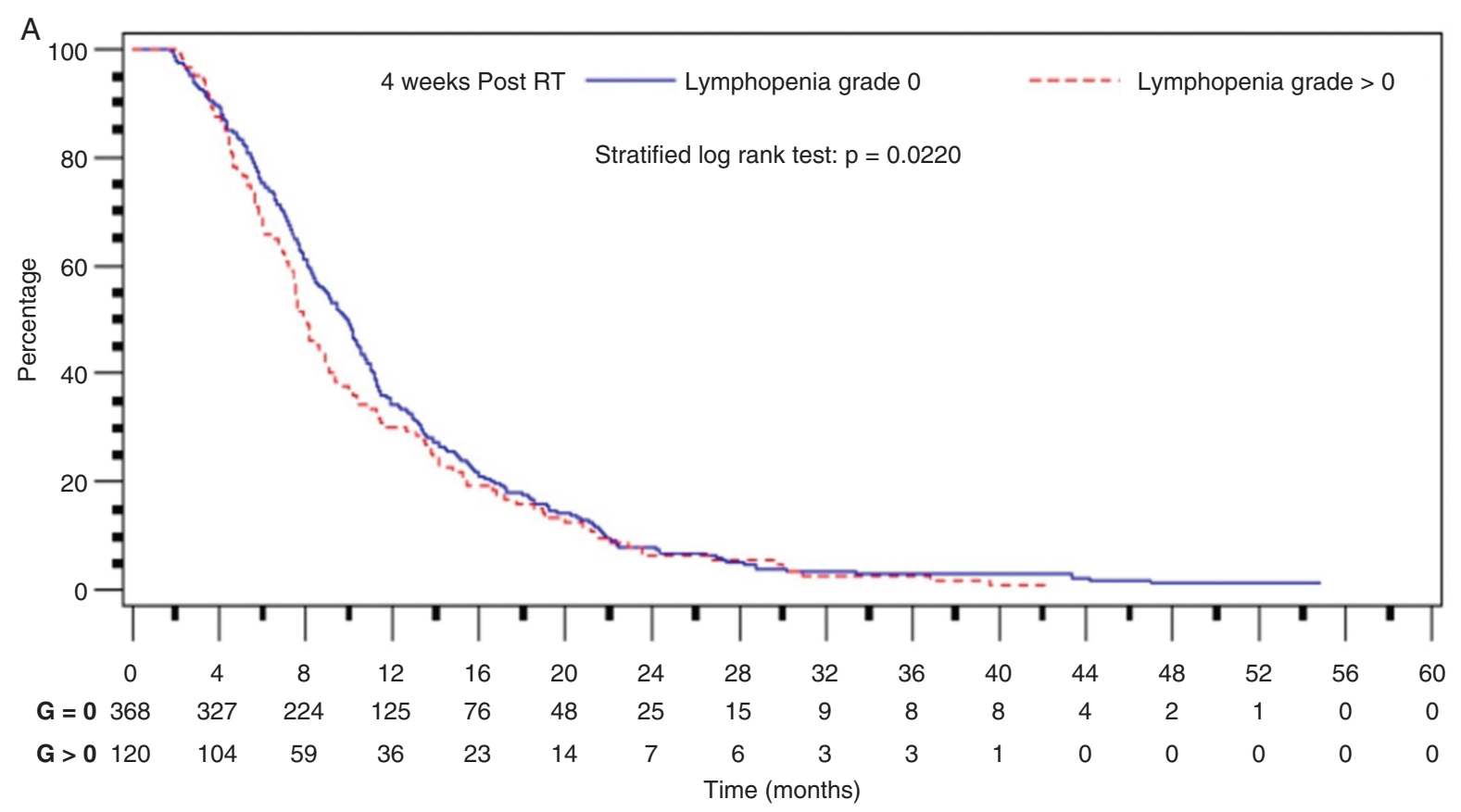

B

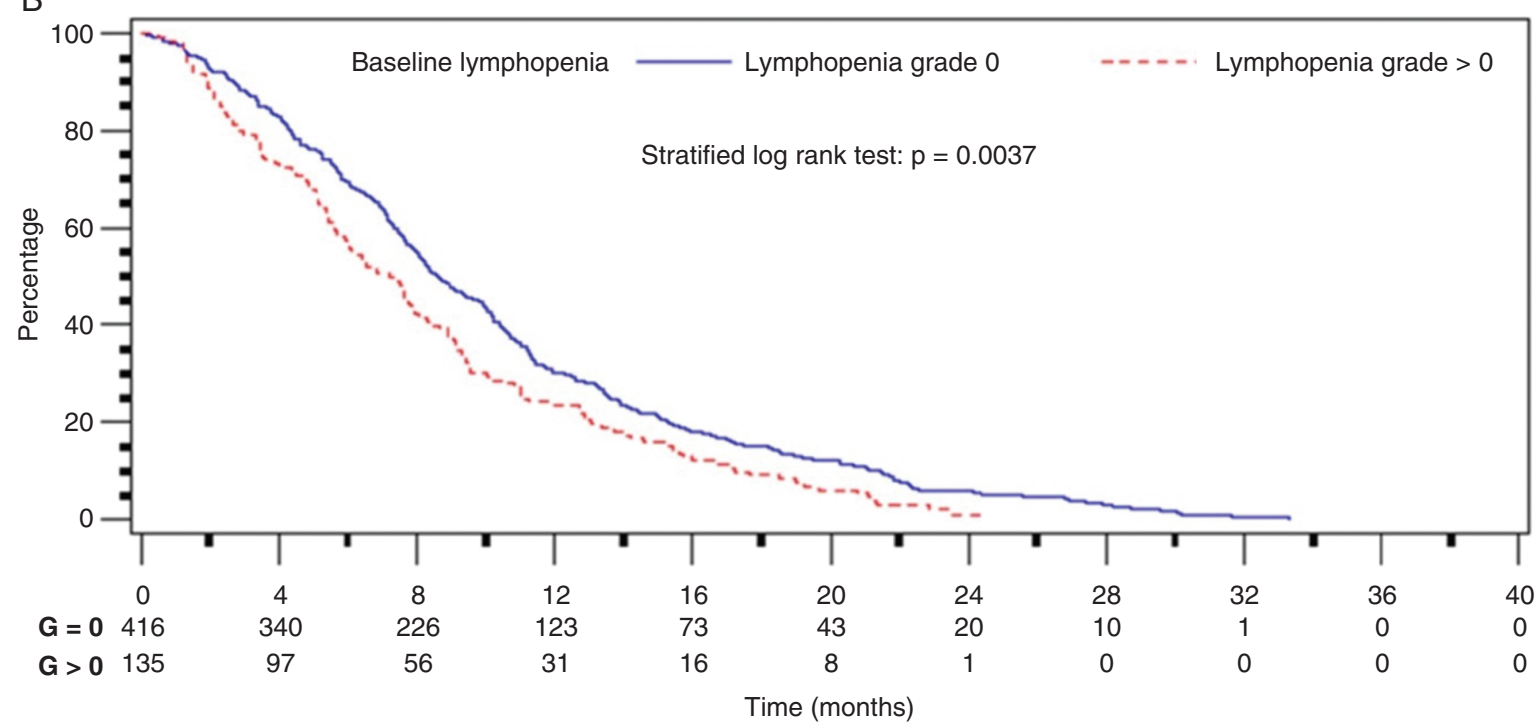

Figure 2. Kaplan-Meier survival curves. (A) Overall survival for 4 weeks post-RT lymphopenia. (B) Overall survival worse with baseline lymphopenia (Grade $>0$ ).

treatment arms. ${ }^{6}$ We showed that baseline lymphopenia significantly increases the risk for developing treatmentrelated lymphopenia, including severe lymphopenia. Therefore, our analysis elucidated an additional facet on the importance of timepoint for lymphopenia, especially at baseline for elderly GBM patients, which had not been previously reported.

Baseline lymphopenia may represent several pretreatment concerns for patients. First, this may serve as a surrogate for poor reserve or immunocompromised patients. Second, this may represent a secondary lymphotoxic effect from steroid exposure prior to trial randomization, which may indicate patients with more clinical symptoms, extensive disease burden, and/or unresectable disease. Analysis on patients enrolled on EORTC trials with breast, non-Hodgkin's lymphoma, and soft tissue sarcoma patients also showed pretreatment lymphocyte count less than $1000 / \mathrm{mm}^{3}$ was associated with higher risk of death. ${ }^{18}$ In GBM patients, pretreatment lymphopenia is associated with post radiation treatment Iymphopenia, ${ }^{19}$ which is consistent with our finding. However, caution should be given for GBM patients with pretreatment lymphopenia 
Table 3. Multivariable Cox Proportional Hazards Model for 4 Weeks Post-RT on OS

\begin{tabular}{|c|c|c|}
\hline Factors & $\mathrm{HR}(95 \% \mathrm{Cl})$ & $P$-value \\
\hline W4 Lymphopenia & & .77 \\
\hline Grade 1 or 2 & $1.09(0.83,1.41)$ & .54 \\
\hline Grade 3 or 4 & $1.14(0.71,1.84)$ & .58 \\
\hline Grade 0 & 1 & \\
\hline \multicolumn{3}{|l|}{ Baseline Lymphopenia } \\
\hline Grade 1+ & $1.30(1.05,1.62)$ & .02 \\
\hline Grade 0 & 1 & \\
\hline \multicolumn{3}{|l|}{ Sex } \\
\hline Male & $1.41(1.16,1.71)$ & .0004 \\
\hline Female & 1 & \\
\hline \multicolumn{3}{|l|}{ Extent of Resection } \\
\hline Biopsy only & $1.59(1.29,1.96)$ & $<.0001$ \\
\hline Partial or complete resection & 1 & \\
\hline \multicolumn{3}{|l|}{ Steroids use } \\
\hline Yes & $1.16(0.93-1.44)$ & .18 \\
\hline No & 1 & \\
\hline \multicolumn{3}{|l|}{ ECOG PS } \\
\hline 2 & $1.14(0.89-1.45)$ & .31 \\
\hline 0 or 1 & 1 & \\
\hline MMSE & $0.976(0.955-0.997)$ & .02 \\
\hline
\end{tabular}

may be due to more extensive tumor and/or dependance of steroids, which may be associated with worse outcome. Although we controlled for steroid exposure at baseline, information regarding the length of exposure and dosing of steroids was not collected. Further study is needed to better understand the impact of steroids on GBM patients' treatment outcomes.

Radiation treatment plan dosimetry has been shown to predict the risk of lymphopenia, and V25 of brain tissue less than $56 \%$ with conventional fractionation regimen may reduce the risk of severe lymphopenia. ${ }^{20}$ Despite the unavailability of detailed dosimetric data on radiation treatment plans in this study, our results show no significant lymphopenia attributable to radiation treatment alone. This may be due to the radiation target volume, and dose fractionation scheduled. Radiation volume is based on residual enhancing tumor and resection cavity, as well as encompassing FLAIR abnormalities. The hypofractionation radiation regimen used in the current trial may further reduce the risk of radiation induced lymphopenia. Based on the estimated probability of circulating lymphocytes through the radiation fields during treatment, the magnitude of radiation exposure and its impact is greatly reduced with hypofractionation. ${ }^{7,21}$

Our current analysis showed short-course RT alone does not lead to lympohpenia after treatment. Development of lymphopenia post-RT is associated with addition of TMZ and baseline lymphopenia. However, only baseline lymphopenia is associated with worse OS regardless of
MGMT status. It may be useful to improve the predication of outcome based on current know risk factor, and help clinical treatment decision making. It may also be an stratification factor for clinical trial design. Further studies are warranted for validation.

\section{Keywords}

elderly | glioblastoma | lymphopenia | short-course radiotherapy | temozolomide

\section{Funding}

This research did not receive any specific grant from funding agencies in the public, commercial, or not-for-profit sectors. The authors would like to Ms. Peggy Grove for philanthropic support for this study.

Authorship Statement. Conception design and development: A.J.S., W.S. Statistics: K.D. Writing and revision of manuscript: A.J.S., W.S. Revision and final approval of manuscript: A.J.S., A.D., I.A., N.J.L., J.P., W.P.M., C.W., C.J.O., J.J.M., A.A.B., C.P., M.F.F., R.N., D.O., J.G.C., W.R., W.W., W.S.

Conflict of interest statement. N.J.L.: Consulting/Advisory Role for Abbvie. Honararia for Merck/Schering Plough. W.P.M.: Expert Testimony for Medcan. R.N.: Consulting/Advisory Role and Expert Testimony for Novocure, Ono Pharmaceutical, Daiichi Sankyo; Honoraria for Chugai Pharma, Eisai, Abbvie, and Nihonkayaku. W.W.: Consulting/Advisory Role for Abbvie, MSD Oncology, and Roche/Genentech. Research funding from Roche, Apogenix, Pfizer. W.S.: Consulting for Brainlab, Varian, Novocure; Research funding for clinical trial: Novocure, Regeneron. The remaining authors have no $\mathrm{COI}$ to declare.

\section{References}

1. Keime-Guibert F, Chinot O, Taillandier L, et al.; Association of FrenchSpeaking Neuro-Oncologists. Radiotherapy for glioblastoma in the elderly. N Engl J Med. 2007;356(15):1527-1535.

2. Roa W, Kepka L, Kumar N, et al. International atomic energy agency randomized phase iii study of radiation therapy in elderly and/or frail patients with newly diagnosed glioblastoma multiforme. J Clin Oncol. 2015;33(35):4145-4150.

3. Roa W, Brasher PM, Bauman G, et al. Abbreviated course of radiation therapy in older patients with glioblastoma multiforme: a prospective randomized clinical trial. J Clin Oncol. 2004;22(9):1583-1588.

4. Wick W, Platten M, Meisner C, et al.; NOA-08 Study Group of Neuro-oncology Working Group (NOA) of German Cancer Society. Temozolomide chemotherapy alone versus radiotherapy alone for 
malignant astrocytoma in the elderly: the NOA-08 randomised, phase 3 trial. Lancet Oncol. 2012;13(7):707-715.

5. Malmström A, Grønberg BH, Marosi C, et al.; Nordic Clinical Brain Tumour Study Group (NCBTSG). Temozolomide versus standard 6-week radiotherapy versus hypofractionated radiotherapy in patients older than 60 years with glioblastoma: the Nordic randomised, phase 3 trial. Lancet Oncol. 2012;13(9):916-926.

6. Perry JR, Laperriere N, O'Callaghan CJ, et al.; Trial Investigators. Shortcourse radiation plus temozolomide in elderly patients with glioblastoma. N Engl J Med. 2017;376(11):1027-1037.

7. Yovino S, Grossman SA. Severity, etiology and possible consequences of treatment-related lymphopenia in patients with newly diagnosed highgrade gliomas. CNS Oncol. 2012;1(2):149-154.

8. Gilbert MR, Wang M, Aldape KD, et al. Dose-dense temozolomide for newly diagnosed glioblastoma: a randomized phase III clinical trial. J Clin Oncol. 2013;31(32):4085-4091.

9. Lee C, Ahn S, Park JS, Song JH, Hong YK, Jeun SS. Effect of cumulative dexamethasone dose during concomitant chemoradiation on lymphopenia in patients with newly diagnosed glioblastoma. Brain Tumor Res Treat. 2020;8(2):71-76.

10. Grossman SA, Ellsworth S, Campian J, et al. Survival in patients with severe lymphopenia following treatment with radiation and chemotherapy for newly diagnosed solid tumors. J Natl Compr Canc Netw. 2015;13(10):1225-1231.

11. Montecino-Rodriguez E, Berent-Maoz B, Dorshkind K. Causes, consequences, and reversal of immune system aging. J Clin Invest. 2013;123(3):958-965.

12. Common Terminology Criteria for Adverse Events (CTCAE). US Department of Health and Human Services, National Institutes of Health, National Cancer Institute.

13. Grossman SA, Ye X, Lesser G, et al.; NABTT CNS Consortium. Immunosuppression in patients with high-grade gliomas treated with radiation and temozolomide. Clin Cancer Res. 2011;17(16):5473-5480.
14. Kim WJ, Dho YS, Ock CY, et al. Clinical observation of lymphopenia in patients with newly diagnosed glioblastoma. I Neurooncol. 2019;143(2):321-328.

15. Byun HK, Kim N, Yoon HI, et al. Clinical predictors of radiation-induced lymphopenia in patients receiving chemoradiation for glioblastoma: clinical usefulness of intensity-modulated radiotherapy in the immunooncology era. Radiat Oncol. 2019;14(1):51.

16. Mendez JS, Govindan A, Leong J, Gao F, Huang J, Campian JL. Association between treatment-related lymphopenia and overall survival in elderly patients with newly diagnosed glioblastoma. J Neurooncol. 2016;127(2):329-335.

17. Grossman SA, Ye X, Piantadosi S, et al.; NABTT CNS Consortium. Survival of patients with newly diagnosed glioblastoma treated with radiation and temozolomide in research studies in the United States. Clin Cancer Res. 2010;16(8):2443-2449.

18. Ray-Coquard I, Cropet C, Van Glabbeke M, et al.; European Organization for Research and Treatment of Cancer Soft Tissue and Bone Sarcoma Group. Lymphopenia as a prognostic factor for overall survival in advanced carcinomas, sarcomas, and lymphomas. Cancer Res. 2009;69(13):5383-5391.

19. Ishikawa E, Yamamoto T, Sakamoto N, et al. Low peripheral lymphocyte count before focal radiotherapy plus concomitant temozolomide predicts severe lymphopenia during malignant glioma treatment. Neurol Med Chir (Tokyo). 2010;50(8):638-644.

20. Huang J, DeWees TA, Badiyan SN, et al. Clinical and dosimetric predictors of acute severe lymphopenia during radiation therapy and concurrent temozolomide for high-grade glioma. Int J Radiat Oncol Biol Phys. 2015;92(5):1000-1007.

21. Kleinberg L, Sloan L, Grossman S, Lim M. Radiotherapy, lymphopenia, and host immune capacity in glioblastoma: a potentially actionable toxicity associated with reduced efficacy of radiotherapy. Neurosurgery. 2019;85(4):441-453. 